\title{
Eliciting Discount Functions when Baseline Consumption changes over Time
}

Anke Gerber

Kirsten I.M. Rohde ${ }^{2}$

1 Hamburg University, Germany;

2 Erasmus School of Economics, Erasmus University Rotterdam, and Tinbergen Institute, the Netherlands. 
Tinbergen Institute is the graduate school and research institute in economics of Erasmus University Rotterdam, the University of Amsterdam and VU University Amsterdam.

More TI discussion papers can be downloaded at http://www.tinbergen.nl

Tinbergen Institute has two locations:

Tinbergen Institute Amsterdam

Gustav Mahlerplein 117

1082 MS Amsterdam

The Netherlands

Tel.: +31(0)205251600

Tinbergen Institute Rotterdam

Burg. Oudlaan 50

3062 PA Rotterdam

The Netherlands

Tel.: +31(0)10 4088900

Fax: $+31(0) 104089031$

Duisenberg school of finance is a collaboration of the Dutch financial sector and universities, with the ambition to support innovative research and offer top quality academic education in core areas of finance.

DSF research papers can be downloaded at: http://www.dsf.nl/

Duisenberg school of finance

Gustav Mahlerplein 117

1082 MS Amsterdam

The Netherlands

Tel.: +31(0)20 5258579 


\title{
Eliciting Discount Functions when Baseline Consumption changes over Time ${ }^{1}$
}

\author{
Anke Gerber ${ }^{2} \quad$ Kirsten I.M. Rohde ${ }^{34}$
}

November 20, 2014

\footnotetext{
${ }^{1}$ The authors would like to thank Peter Wakker and Jawwad Noor for helpful comments and suggestions.

${ }^{2}$ Department of Economics, Hamburg University, Von-Melle-Park 5, 20146 Hamburg, Germany, e-mail: anke.gerber@wiso.uni-hamburg.de

${ }^{3}$ Department of Economics, H13-25, Erasmus University, P.O. Box 1738, 3000 DR Rotterdam, the Netherlands, e-mail: rohde@ese.eur.nl

${ }^{4}$ Tinbergen Institute and Erasmus Research Institute of Management
} 


\begin{abstract}
Many empirical studies on intertemporal choice report preference reversals in the sense that a preference between a small reward to be received soon and a larger reward to be received later reverses as both rewards are equally delayed. Such preference reversals are commonly interpreted as contradicting constant discounting. This interpretation is correct only if baseline consumption to which the outcomes are added, remains constant over time. The difficulty with measuring discounting when baseline consumption changes over time, is that delaying an outcome has two effects: (1) due to the change in baseline consumption, it changes the extra utility from receiving the outcome, and (2) it changes the factor by which this extra utility is discounted. In this paper we propose a way to disentangle the two effects, which allows us to draw conclusions about discounting even when baseline consumption changes over time.
\end{abstract}

Keywords: Hyperbolic discounting; Constant discounting; Preference reversals; Decreasing impatience

JEL classification: D91, D81 


\section{Introduction}

Many empirical studies show that people's choice behavior is time inconsistent in the sense that a preference between a small reward to be received soon and a larger reward to be received later, reverses as both rewards are equally delayed. This preference reversal is commonly interpreted as contradicting Samuelson's (1937) constant discounting and as evidence for hyperbolic discounting (Frederick et al., 2002). We show that this interpretation is not justified if the decision maker adds rewards to a baseline consumption level which may change over time in a manner unknown to the experimenter. Noor (2009) showed that another common approach to falsify constant discounting only works if baseline consumption is constant over time. This is bad news. Unless we know that baseline consumption remains unchanged over time, we cannot draw clear conclusions about the discount function from the usual intertemporal choices that are observed in the literature.

This paper proposes an approach to measure properties of the discount function when it is unknown how baseline consumption may change over time. We provide conditions under which one can conclude that choice behavior is inconsistent with constant discounting, even when one does not know how baseline consumption changes over time.

The difficulty with measuring discounting when baseline consumption changes over time, is that delaying an outcome has two effects: (1) due to the change in baseline consumption, it changes the extra utility from receiving the outcome, and (2) it changes the factor by which this extra utility is discounted. In order to draw conclusions about discounting one needs to disentangle these two effects, which seems impossible at first sight. The main contribution of this paper is to provide a method for disentangling the two effects.

Key to our approach is to extend the domain of preferences from dated outcomes $(t: x)$ that yield outcome $x$ for sure at date $t$, to risky dated outcomes $(t: p: x)$ that yield outcome $x$ with probability $p$ at date $t$. We consider tradeoffs between delaying an outcome and making the outcome more risky by determining probability equivalents of delays. Imagine we start with the receipt of an outcome $x$ at date 0 and we delay it to date 
$t$. Then we determine the probability $p$ that would make 'receiving the outcome at date $t$ for sure' equivalent to 'receiving the outcome at date 0 with probability $p$ '. There are only few studies (Keren and Roelofsma, 1995, Baucells and Heukamp, 2012, and Gerber and Rohde, 2014) that analyze the tradeoff between probability and delay. Our results show that analyzing this tradeoff by extending the preference domain to risky dated outcomes, may prove helpful when preferences over dated outcomes alone are not sufficient to draw conclusions about the discount function.

\section{Baseline Consumption and Impatience}

Let $\mathcal{T}=\{0,1, \ldots, T\}$ be the set of dates, where date 0 represents today and date $T \in \mathbb{N}$ is the decision maker's time horizon. ${ }^{1}$ The decision maker (DM) evaluates risky dated outcomes $(t: p: x)$, which give outcome $x \in \mathbb{R}_{+}$with probability $p \in[0,1]$ at date $t \in \mathcal{T}$ and nothing otherwise. We assume that the DM's preference relation $\succcurlyeq$ over risky dated outcomes $\mathcal{D}=\mathcal{T} \times[0,1] \times \mathbb{R}_{+}$can be represented by the utility function $V: \mathcal{D} \rightarrow \mathbb{R}$ with

$$
V(t: p: x)=p \delta(t)\left[u\left(b_{t}+x\right)-u\left(b_{t}\right)\right]
$$

where $u: \mathbb{R}_{+} \rightarrow \mathbb{R}$ is a strictly increasing, strictly concave, and twice continuously differentiable utility function, where $\delta: \mathcal{T} \rightarrow(0,1]$ is a strictly decreasing discount function with $\delta(0)=1$, and where $b_{t} \in \mathbb{R}_{+}$is the DM's baseline consumption at date $t$. Hence, whenever the DM receives an outcome $x$ at date $t$, he adds it to his baseline consumption and consumes the sum of both, which yields utility $u\left(b_{t}+x\right)$. If $x$ is received with probability $p$ at date $t$, the increase in expected utility at date $t$ therefore is $p\left[u\left(b_{t}+x\right)-u\left(b_{t}\right)\right]$, which is equivalent to an increase in expected utility of $\delta(t) p\left[u\left(b_{t}+x\right)-u\left(b_{t}\right)\right]$ at date 0 . Thus, baseline consumption affects the extra utility generated by the receipt of an outcome $x$.

This model is a special case of the model we characterized in Gerber and Rohde (2014)

\footnotetext{
${ }^{1}$ In order to avoid technicalities we assume $T$ to be finite.
} 
with weighting function $w(p, t)=p \delta(t) .^{2,3,4}$ We assume that baseline consumption is riskless, but, as in Noor (2009), it can be viewed as a "stand-in" for any other factors that possibly affect marginal utility.

The DM has decreasing (constant, increasing) absolute risk aversion if $-u^{\prime \prime}(x) / u^{\prime}(x)$ is decreasing (constant, increasing) in $x$.

A preference reversal typically observed in empirical studies is

$$
(0: 1: x) \sim(\tau: 1: y) \text { and }(t: 1: x) \prec(t+\tau: 1: y)
$$

with $y>x>0$, and $\tau, t>0$, i.e. the DM is indifferent between receiving $y$ with probability 1 at date $\tau$ and receiving the smaller payoff $x$ with probability 1 at date 0 , but he strictly prefers to wait for the larger payoff $y$ if both rewards are delayed by $t$. Based on this preference reversal it is commonly concluded that the discount function cannot be the exponential $\delta(t)=e^{-r t}$. We will show, though, that this conclusion is only justified if baseline consumption is constant over time.

In line with Prelec $(1989,2004)$ we say that decreasing impatience holds when the near future is discounted at a higher rate than the far future.

Definition 2.1 Decreasing (constant, increasing) impatience holds if for all $s<t$ and for all $\tau>0$

$$
\frac{\delta(s)}{\delta(s+\tau)}>(=,<) \frac{\delta(t)}{\delta(t+\tau)}
$$

Constant impatience is equivalent to the discount function being exponential, i.e. $\delta(t)=$

\footnotetext{
${ }^{2}$ In Gerber and Rohde (2014) we let $\mathcal{T}=\mathbb{R}_{+}$.

${ }^{3}$ All results in Sections 2 and 3 remain valid if we would have $V(t: p: x)=w(p) \delta(t)\left[u\left(b_{t}+x\right)-u\left(b_{t}\right)\right]$ instead, with $w$ increasing and non-linear.

${ }^{4}$ This representation of a preference relation over $\mathcal{D}$ is obtained if the DM's preference relation $\succsim$ over consumption streams $\left(\tilde{c}_{0}, \tilde{c}_{1}, \ldots, \tilde{c}_{T}\right)$, where $\tilde{c}_{t}$ is a random variable with realizations in $\mathbb{R}_{+}$, is represented by a discounted expected utility function, i.e. $\left(\tilde{c}_{0}, \ldots, \tilde{c}_{T}\right) \succsim\left(\tilde{c}_{0}^{\prime}, \ldots, \tilde{c}_{T}^{\prime}\right) \Longleftrightarrow \sum_{t} \delta(t) E U\left(\tilde{c}_{t}\right) \geq$ $\sum_{t} \delta(t) E U\left(\tilde{c}_{t}^{\prime}\right)$, where $E U(\cdot)$ is expected utility taken with respect to some von Neumann-Morgenstern utility function $u$.
} 
$e^{-r t}$ for some $r>0$. Decreasing impatience is satisfied for the generalized hyperbolic discount function $\delta(t)=(1+\alpha t)^{-\beta / \alpha}$, with $\alpha, \beta>0$ (Loewenstein and Prelec, 1992), and increasing impatience holds for the constant relative decreasing impatience discount function $\delta(t)=e^{-r t^{1-d}}$ with $d<0$ and $r>0$ (Bleichrodt et al., 2009).

If baseline consumption is constant over time, then preferences of type (2) contradict both increasing and constant impatience. To see this, observe that (2) is equivalent to

$$
\begin{aligned}
\delta(\tau) & =\frac{u\left(b_{0}+x\right)-u\left(b_{0}\right)}{u\left(b_{\tau}+y\right)-u\left(b_{\tau}\right)} \quad \text { and } \\
\frac{\delta(t+\tau)}{\delta(t)} & >\frac{u\left(b_{t}+x\right)-u\left(b_{t}\right)}{u\left(b_{t+\tau}+y\right)-u\left(b_{t+\tau}\right)} .
\end{aligned}
$$

If baseline consumption is constant over time then this implies

$$
\delta(\tau)<\frac{\delta(t+\tau)}{\delta(t)}
$$

which contradicts both increasing and constant impatience. Similarly, if baseline consumption is constant, then

$$
(0: 1: x) \sim(\tau: 1: y) \text { and }(t: 1: x) \succ(t+\tau: 1: y)
$$

contradicts both decreasing and constant impatience. However, if baseline consumption changes over time, then (2) need not contradict increasing or constant impatience as can be demonstrated with the following example:

Example 2.2 Let $u(x)=-e^{-r x}$ for all $x$, where $r>0$ is the constant absolute risk aversion parameter. Moreover, let $b_{t}=b_{0} \sqrt{t}$ for some $b_{0}>0$. Then there exists a discount function $\delta$ with increasing or constant impatience such that (2) is satisfied. To see this, observe that for the given utility function and $\tau, t>0, y>x>0$,

$$
\begin{aligned}
\frac{u\left(b_{t}+x\right)-u\left(b_{t}\right)}{u\left(b_{t+\tau}+y\right)-u\left(b_{t+\tau}\right)} & <\frac{u\left(b_{0}+x\right)-u\left(b_{0}\right)}{u\left(b_{\tau}+y\right)-u\left(b_{\tau}\right)} \\
\Longleftrightarrow e^{-r\left(b_{t}-b_{t+\tau}\right)} & <e^{-r\left(b_{0}-b_{\tau}\right)} \\
\Longleftrightarrow b_{t}-b_{t+\tau} & >b_{0}-b_{\tau}
\end{aligned}
$$


where the latter is satisfied for the given baseline consumption. If we choose $\delta$ such that

$$
\delta(\tau)=\frac{u\left(b_{0}+x\right)-u\left(b_{0}\right)}{u\left(b_{\tau}+y\right)-u\left(b_{\tau}\right)}
$$

and

$$
\frac{u\left(b_{0}+x\right)-u\left(b_{0}\right)}{u\left(b_{\tau}+y\right)-u\left(b_{\tau}\right)}>\frac{\delta(t+\tau)}{\delta(t)}>\frac{u\left(b_{t}+x\right)-u\left(b_{t}\right)}{u\left(b_{t+\tau}+y\right)-u\left(b_{t+\tau}\right)}
$$

then (2) is satisfied and $\delta$ (locally) satisfies increasing impatience. If instead we choose $\delta$ such that

$$
\delta(\tau)=\frac{\delta(t+\tau)}{\delta(t)}=\frac{u\left(b_{0}+x\right)-u\left(b_{0}\right)}{u\left(b_{\tau}+y\right)-u\left(b_{\tau}\right)},
$$

then (2) is satisfied and $\delta$ (locally) satisfies constant impatience.

Similarly, if we do not know how baseline consumption changes over time, then (5) need not contradict decreasing or constant impatience. Hence, summarizing we have the following result:

\section{Theorem 2.3}

(i) If baseline consumption is constant over time, i.e. $b_{t}=b_{0}$ for all $t$, then (2) contradicts both increasing and constant impatience, and (5) contradicts both decreasing and constant impatience.

(ii) If baseline consumption changes over time, then both (2) and (5) are consistent with decreasing, constant, and increasing impatience.

In Gerber and Rohde (2010) we showed similar results for the specific case where baseline consumption at any date $t$ is a mean-preserving spread of baseline consumption at date $t-1$. These results are in the same spirit as Noor's (2009) finding that, when baseline consumption is not constant, properties of the money-discount function do not necessarily translate into properties of the discount function. Noor (2009) dealt with another common approach used in the literature to verify whether discount functions satisfy decreasing, 
increasing, or constant impatience. If $\psi(x, t)$ is the net present value of outcome $x$ at date $t$, i.e. $(0: 1: \psi(x, t)) \sim(t: 1: x)$, then many studies in the literature take the moneydiscount function $\phi^{x}(t)=\psi(x, t) / x$ as (an approximation of) the discount function $\delta(t)$, which, as Noor shows, is only justified for small $x$ if baseline consumption is approximately constant.

In the next section we will show how we can test for decreasing, constant, or increasing impatience, without knowing how baseline consumption evolves.

\section{A Test for Constant Impatience}

In the following we will introduce an approach which does allow us to draw conclusions about the mentioned properties of the discount function, even if the DM's baseline consumption is not observed. Key to our approach is the determination of probabilities that are experienced as being equivalent to a particular delay of some payoff.

Consider a DM who receives a reward $x$ at date $\tau$ for sure. Now assume that the receipt of this reward will either be delayed to date $t$ or will be received at date $\tau$ with a lower probability $p<1$. If the DM is indifferent between the delay and the probability, i.e. if

$$
(\tau: p: x) \sim(t: 1: x)
$$

then we say that $p$ is the probability equivalent of the delay from $\tau$ to $t$ for outcome $x$. First we show that under decreasing and increasing absolute risk aversion the probability equivalents of a delay from $\tau$ to $t$ for two outcomes $x<y$ allow us to determine whether baseline consumption is increasing, constant, or decreasing during the delay.

Theorem 3.1 Let $t>\tau \geq 0$ and $y>x>0$ and let $p$ and $p^{\prime}$ be defined by

$$
(\tau: p: x) \sim(t: 1: x) \text { and }\left(\tau: p^{\prime}: y\right) \sim(t: 1: y)
$$

Then the following holds true:

(i) If the DM satisfies decreasing absolute risk aversion, then

$$
b_{t}>(=<) b_{\tau} \Longleftrightarrow p^{\prime}>(=<) p .
$$


(ii) If the DM satisfies increasing absolute risk aversion, then

$$
b_{t}>(=<) b_{\tau} \Longleftrightarrow p^{\prime}<(=>) p
$$

(iii) If the DM satisfies constant absolute risk aversion, then $p^{\prime}=p$, independently of $b_{\tau}$ and $b_{t}$.

Hence, under the assumption that the DM either satisfies decreasing or increasing absolute risk aversion, from observing the ratio $p / p^{\prime}$ of probability equivalents of a delay from $\tau$ to $t$ for two outcomes $y>x$, we can conclude whether the DM perceives baseline consumption at $t$ to be larger, equal, or smaller than baseline consumption at $\tau$. It remains to be verified how plausible the assumption of decreasing or increasing absolute risk aversion is. There are two ways one can approach this. Firstly, we can check how the DM's absolute risk aversion changes with income, either directly by measuring the DM's utility function $u$ for payoffs to be received at date 0 (cf. Section 4), or indirectly by measuring the risk premium associated with a simple binary lottery for different upfront payments that change the DM's baseline consumption at date 0. Secondly, we can rely on considerable empirical evidence for decreasing absolute risk aversion (see, e.g. Donkers et al., 2001; Hartog et al., 2002; Lee, 2008) and assume this property of the DM's utility function without actually testing for it.

Theorem 3.1 allows us to derive testable hypotheses concerning deviations from constant impatience:

Theorem 3.2 Let $s \geq 0$ and let $y>x>0$ and $\tau$ be such that

$$
(s: 1: x) \sim(s+\tau: 1: y) .
$$

Let $t>s$ and $Y>X>0$ and $p, p^{\prime}, q, q^{\prime}$ be such that

$$
\begin{aligned}
(s: p: X) \sim(t: 1: X) & \& \quad\left(s: p^{\prime}: Y\right) \sim(t: 1: Y), \text { and } \\
(s+\tau: q: X) \sim(t+\tau: 1: X) \quad \& \quad\left(s+\tau: q^{\prime}: Y\right) & \sim(t+\tau: 1: Y) .
\end{aligned}
$$


(i) Let the DM satisfy decreasing absolute risk aversion. Then

$$
(t: 1: x) \prec(t+\tau: 1: y) \& p^{\prime}<p \& q^{\prime}>q
$$

contradicts both increasing and constant impatience and

$$
(t: 1: x) \succ(t+\tau: 1: y) \& p^{\prime}>p \& q^{\prime}<q
$$

contradicts both decreasing and constant impatience.

(ii) Let the DM satisfy increasing absolute risk aversion. Then

$$
(t: 1: x) \prec(t+\tau: 1: y) \& p^{\prime}>p \& q^{\prime}<q
$$

contradicts both increasing and constant impatience and

$$
(t: 1: x) \succ(t+\tau: 1: y) \& p^{\prime}<p \& q^{\prime}>q
$$

contradicts both decreasing and constant impatience.

(iii) If the DM satisfies constant absolute risk aversion, then $p^{\prime}=p$ and $q^{\prime}=q$ and we do not have sufficient information to determine whether the discount function satisfies decreasing, increasing, or constant impatience.

Theorem 3.1 showed that probability equivalents of delays can be used to determine whether baseline consumption is increasing, constant, or decreasing, which, by the proof of Theorem 3.2, can provide sufficient information to reject constant, decreasing, or increasing impatience. More precisely, in the first part of Theorem 3.2 (i) we show that under decreasing absolute risk aversion, if baseline consumption is decreasing from date $s$ to date $t$ and increasing from date $s+\tau$ to date $t+\tau$, then $(s: 1: x) \sim(s+\tau: 1: y)$ and $(t: 1: x) \prec(t+\tau: 1: y)$ contradict both increasing and constant impatience. The same preferences may, but need not, contradict both increasing and constant impatience if baseline consumption is increasing throughout. Thus, Theorem 3.2 allows us to detect some cases in which both constant and increasing or both constant and decreasing impatience are falsified, but not all. 
One way to detect all cases that falsify both constant and increasing or both constant and decreasing impatience, is to measure the discount function entirely. If we do not only want to know whether impatience is constant, decreasing, or increasing, but want to measure the precise discount factor for each date, then we will not only need to know whether baseline consumption increases or decreases, but also by exactly how much it increases or decreases. In the next section we will show how this can be done.

\section{Eliciting the Discount Function}

In this section we will illustrate how the discount function can be elicited when baseline consumption may change over time. Without loss of generality we normalize utility such that $u\left(b_{0}\right)=0$ and $u\left(b_{0}+M\right)=1$ for some $M>0$. Throughout this section we will also assume that we know the utility function $\tilde{u}(x)=u\left(b_{0}+x\right)$. This function can be measured as follows. For every outcome $x<M$ we elicit $p_{x}$ such that $\left(0: p_{x}: M\right) \sim(0: 1: x)$, which yields $u\left(b_{0}+x\right)=p_{x} u\left(b_{0}+M\right)=p_{x}$. For every outcome $x>M$ we elicit $p_{x}$ such that $\left(0: p_{x}: x\right) \sim(0: 1: M)$, which yields $u\left(b_{0}+x\right)=u\left(b_{0}+M\right) / p_{x}=1 / p_{x}$.

In order to elicit the discount function we need to investigate tradeoffs between receiving different outcomes at different dates. The usual approach to elicit the discount function is to establish indifferences of the form

$$
(0: 1: x) \sim(t: 1: y)
$$

which yields

$$
u\left(b_{0}+x\right)=\delta(t)\left[u\left(b_{t}+y\right)-u\left(b_{t}\right)\right]
$$

If baseline consumption does not change over time, then knowing how $u\left(b_{0}+x\right)$ changes with $x$, is sufficient to extract $\delta(t)$ from (8). If we do not know how baseline consumption changes over time, i.e. if we do not know $b_{t}$, then we need more information to extract $\delta(t)$ from (8).

The following theorem shows that substituting indifference (7) by the two indifferences

$$
(0: p: x) \sim(t: 1: x) \text { and }\left(0: p^{\prime}: y\right) \sim(t: 1: y)
$$


gives us sufficient information to determine $\delta(t)$ if the DM satisfies decreasing or increasing absolute risk aversion.

Theorem 4.1 Let $t>0$ and $y>x>0$ and let $p$ and $p^{\prime}$ be defined by

$$
(0: p: x) \sim(t: 1: x) \quad \text { and } \quad\left(0: p^{\prime}: y\right) \sim(t: 1: y)
$$

If the decision maker satisfies decreasing or increasing absolute risk aversion, then $b_{t}-b_{0}$ and $\delta(t)$ are uniquely determined.

\section{Discussion}

Many intertemporal decision problems in economics and finance build on the discounted expected utility model where individuals evaluate a future reward by discounting the extra utility this rewards generates on top of baseline consumption. Several empirical studies have challenged the standard assumption of constant discounting in this model and have provided evidence for preference reversals that seem to be inconsistent with constant discounting. However, as we point out in this paper, these studies have only rejected the joint hypothesis that the DM's baseline consumption is constant and that discounting is constant over time. Neglecting the effect of changes in baseline consumption on intertemporal choice behavior can lead to biased estimates of a DM's discount function. In order to avoid this bias one has to disentangle the effect that delaying an outcome has on discounting on the one hand and on the DM's marginal utility on the other hand, where the latter is to a large extent determined by baseline consumption. At first sight, it seems impossible to disentangle the two effects in order to derive properties of the discount function. Yet, we showed that it is possible to draw conclusions about discounting, even when the change in baseline consumption is unknown.

We demonstrated how the change in baseline consumption and the discount function can both be determined by eliciting the probability equivalents of a delay for two different outcomes. We suggest not only a new way to measure discounting, but also a revealed preference approach to elicit the DM's perceived future baseline consumption. This approach, 
by which a DM reveals his future baseline consumption through the probability equivalents of payoff delays, is clearly preferable to directly asking a DM for his expectations about future consumption, since the latter would not give him an incentive to truthfully report his future consumption. Thus, extending the preference domain from dated outcomes to risky dated outcomes turned out to be useful for analyzing intertemporal choice.

\section{Appendix}

Proof of Theorem 3.1: $(\tau: p: x) \sim(t: 1: x)$ holds if and only if

$$
p \delta(\tau)\left[u\left(b_{\tau}+x\right)-u\left(b_{\tau}\right)\right]=\delta(t)\left[u\left(b_{t}+x\right)-u\left(b_{t}\right)\right]
$$

Similarly, $\left(\tau: p^{\prime}: y\right) \sim(t: 1: y)$ holds if and only if

$$
p^{\prime} \delta(\tau)\left[u\left(b_{\tau}+y\right)-u\left(b_{\tau}\right)\right]=\delta(t)\left[u\left(b_{t}+y\right)-u\left(b_{t}\right)\right]
$$

From (9) and (10) it follows that

$$
\frac{p}{p^{\prime}}=\frac{\left[u\left(b_{t}+x\right)-u\left(b_{t}\right)\right]\left[u\left(b_{\tau}+y\right)-u\left(b_{\tau}\right)\right]}{\left[u\left(b_{t}+y\right)-u\left(b_{t}\right)\right]\left[u\left(b_{\tau}+x\right)-u\left(b_{\tau}\right)\right]}
$$

Clearly, if $b_{t}=b_{\tau}$, then $p^{\prime}=p$ independent of the DM's degree of risk aversion.

Assume decreasing absolute risk aversion. Then for all $z<x$ and all $b$ we have

$$
\begin{aligned}
& -\frac{u^{\prime \prime}(b+x)}{u^{\prime}(b+x)}<-\frac{u^{\prime \prime}(b+z)}{u^{\prime}(b+z)} \\
\Longleftrightarrow & u^{\prime \prime}(b+x) u^{\prime}(b+z)>u^{\prime \prime}(b+z) u^{\prime}(b+x) \\
\Longrightarrow & \int_{0}^{x} u^{\prime \prime}(b+x) u^{\prime}(b+z) d z>\int_{0}^{x} u^{\prime \prime}(b+z) u^{\prime}(b+x) d z \\
\Longleftrightarrow & {[u(b+x)-u(b)] u^{\prime \prime}(b+x)>\left[u^{\prime}(b+x)-u^{\prime}(b)\right] u^{\prime}(b+x) } \\
\Longleftrightarrow & \frac{u^{\prime \prime}(b+x)}{u^{\prime}(b+x)}>\frac{u^{\prime}(b+x)-u^{\prime}(b)}{u(b+x)-u(b)} .
\end{aligned}
$$

It follows that

$$
\frac{d}{d x} \frac{u^{\prime}(b+x)-u^{\prime}(b)}{u(b+x)-u(b)}>0
$$


which implies

$$
\frac{u^{\prime}(b+x)-u^{\prime}(b)}{u(b+x)-u(b)}<\frac{u^{\prime}(b+y)-u^{\prime}(b)}{u(b+y)-u(b)} .
$$

It follows that

$$
\frac{d}{d b}\left[\frac{u(b+x)-u(b)}{u(b+y)-u(b)}\right]<0
$$

Thus,

$$
b_{t}>(=<) b_{\tau}
$$

if and only if

$$
\frac{u\left(b_{\tau}+x\right)-u\left(b_{\tau}\right)}{u\left(b_{\tau}+y\right)-u\left(b_{\tau}\right)}>(=<) \frac{u\left(b_{t}+x\right)-u\left(b_{t}\right)}{u\left(b_{t}+y\right)-u\left(b_{t}\right)}
$$

if and only if

$$
p^{\prime}>(=<) p
$$

With increasing absolute risk aversion the inequalities are always the reverse, and with constant absolute risk aversion the inequalities are equalities, which proves the result.

Proof of Theorem 3.2: From $(s: 1: x) \sim(s+\tau: 1: y)$ we know that

$$
\frac{\delta(s)}{\delta(s+\tau)}=\frac{u\left(b_{s+\tau}+y\right)-u\left(b_{s+\tau}\right)}{u\left(b_{s}+x\right)-u\left(b_{s}\right)} .
$$

Consider first the case where $(t: 1: x) \prec(t+\tau: 1: y)$. This is equivalent to

$$
\frac{\delta(t)}{\delta(t+\tau)}<\frac{u\left(b_{t+\tau}+y\right)-u\left(b_{t+\tau}\right)}{u\left(b_{t}+x\right)-u\left(b_{t}\right)}
$$

Suppose that $b_{t}<b_{s}$ and $b_{t+\tau}>b_{s+\tau}$. Then

$$
\frac{\delta(s)}{\delta(s+\tau)}=\frac{u\left(b_{s+\tau}+y\right)-u\left(b_{s+\tau}\right)}{u\left(b_{s}+x\right)-u\left(b_{s}\right)}>\frac{u\left(b_{t+\tau}+y\right)-u\left(b_{t+\tau}\right)}{u\left(b_{t}+x\right)-u\left(b_{t}\right)}>\frac{\delta(t)}{\delta(t+\tau)}
$$

Thus, $b_{t}<b_{s}$ and $b_{t+\tau}>b_{s+\tau}$ is only consistent with decreasing impatience. Similarly, $(t: 1: x) \succ(t+\tau: 1: y)$ together with $b_{t}>b_{s}$ and $b_{t+\tau}<b_{s+\tau}$ is only consistent with increasing impatience.

From Theorem 3.1 we know that under decreasing absolute risk aversion $b_{t}<b_{s}$ and $b_{t+\tau}>b_{s+\tau}$ hold if and only if $p^{\prime}<p$ and $q^{\prime}>q$. Similarly, under decreasing absolute 
risk aversion $b_{t}>b_{s}$ and $b_{t+\tau}<b_{s+\tau}$ hold if and only if $p^{\prime}>p$ and $q^{\prime}<q$. Moreover, by Theorem 3.1 under increasing absolute risk aversion $b_{t}<b_{s}$ and $b_{t+\tau}>b_{s+\tau}$ hold if and only if $p^{\prime}>p$ and $q^{\prime}<q$, and $b_{t}>b_{s}$ and $b_{t+\tau}<b_{s+\tau}$ hold if and only if $p^{\prime}<p$ and $q^{\prime}>q$.

Theorem 3.1 also implies that, under constant absolute risk aversion, $p^{\prime}=p$ and $q^{\prime}=q$ independent of $b_{s}, b_{t}, b_{s+\tau}$ and $b_{t+\tau}$. Therefore, from observing $p$ and $q$ we cannot conclude anything about the DM's baseline consumption and hence about the discount function.

Proof of Theorem 4.1: Define the function $\tilde{u}(x)=u\left(b_{0}+x\right)$. Note that if $u$ satisfies decreasing (increasing) absolute risk aversion, then $\tilde{u}$ satisfies decreasing (increasing) absolute risk aversion.

We have

$$
\begin{aligned}
u\left(b_{t}+x\right)-u\left(b_{t}\right)=u\left(b_{0}+b_{t}-b_{0}+x\right)-u\left(b_{0}+b_{t}-\right. & \left.b_{0}\right) \\
& =\tilde{u}\left(b_{t}-b_{0}+x\right)-\tilde{u}\left(b_{t}-b_{0}\right) .
\end{aligned}
$$

Then Eq.(11) with $\tau=0$ can be rewritten as

$$
\frac{p}{p^{\prime}}=\frac{\left[\tilde{u}\left(b_{t}-b_{0}+x\right)-\tilde{u}\left(b_{t}-b_{0}\right)\right][\tilde{u}(y)]}{\left[\tilde{u}\left(b_{t}-b_{0}+y\right)-\tilde{u}\left(b_{t}-b_{0}\right)\right][\tilde{u}(x)]}
$$

From inequality (16) in the proof of Theorem 3.1 there is exactly one $c_{t}=b_{t}-b_{0}$ which satisfies Eq.(22). Since we know $\tilde{u}$, we have everything we need to determine $c_{t}$.

We also know that

$$
\delta(t)=p \frac{u\left(b_{0}+x\right)-u\left(b_{0}\right)}{u\left(b_{t}+x\right)-u\left(b_{t}\right)}=p \frac{\tilde{u}(x)}{\tilde{u}\left(b_{t}-b_{0}+x\right)-\tilde{u}\left(b_{t}-b_{0}\right)},
$$

which is then uniquely defined. 


\section{References}

Baucells, Manel, and Franz H. Heukamp (2012), "Probability and Time Trade-Off," Management Science, 58, 831-842.

Bleichrodt, Han, Kirsten I.M. Rohde, and Peter P. Wakker (2009), "Non-Hyperbolic Time Inconsistency," Games and Economic Behavior, 66, 27-38.

Donkers, Bas, Bertrand Melenberg, and Arthur van Soest (2001), "Estimating Risk Attitudes using Lotteries: A Large Sample Approach," Journal of Risk and Uncertainty, 22, 165-195.

Frederick, Shane, George Loewenstein, and Ted O’Donoghue (2002), “Time Discounting and Time Preference: a Critical Review," Journal of Economic Literature, 40, 351-401.

Gerber, Anke, and Kirsten I.M. Rohde (2010), "Risk and Preference Reversals in Intertemporal Choice," Journal of Economic Behavior ES Organization, 76, 654-668.

Gerber, Anke, and Kirsten I.M. Rohde (2014), "Weighted Temporal Utility," Tinbergen Institute Discussion Paper.

Hartog, Joop, Ada Ferrer-i-Carbonell, and Nicole Jonker (2002), "Linking Measured Risk Aversion to Individual Characteristics," Kyklos, 55, 3-26.

Keren, Gideon B., and Peter H.M.P. Roelofsma (1995), "Immediacy and certainty in intertemporal choice," Organizational Behavior and Human Decision Processes, 63, 287297.

Lee, Jinkwon (2008), "The Effect of Background Risk in a Simple Chance Improving Decision Model," Journal of Risk and Uncertainty, 36, 19-41.

Loewenstein, George, and Drazen Prelec (1992), "Anomalies in intertemporal choice: evidence and an interpretation, Quarterly Journal of Economics, 107, 573-597.

Noor, Jawwad (2009), "Hyperbolic Discounting and the Standard Model: Eliciting Discount Functions," Journal of Economic Theory, 144, 2077-2083.

Prelec, Drazen (1989), "Decreasing Impatience: Definition and Consequences," Working Paper. 
Prelec, Drazen (2004), "Decreasing Impatience: A Criterion for Non-stationary Time Preference and 'Hyperbolic' Discounting," Scandinavian Journal of Economics, 106, 511532.

Samuelson, Paul A. (1937), "A note on measurement of utility," The Review of Economic Studies, 4, 155-161. 